Case Report

\title{
Effects of Mifepristone on Nonalcoholic Fatty Liver Disease in a Patient with a Cortisol-Secreting Adrenal Adenoma
}

\author{
Enzo Ragucci, ${ }^{1}$ Dat Nguyen, ${ }^{2}$ Michele Lamerson, ${ }^{2}$ and Andreas G. Moraitis ${ }^{2}$ \\ ${ }^{1}$ Diabetes and Endocrinology Consultants, 2 Crosfield Ave, No. 204, West Nyack, NY 10994, USA \\ ${ }^{2}$ Corcept Therapeutics, 149 Commonwealth Drive, Menlo Park, CA 94025, USA \\ Correspondence should be addressed to Andreas G. Moraitis; amoraitis@corcept.com
}

Received 21 June 2017; Accepted 23 October 2017; Published 19 November 2017

Academic Editor: John Broom

Copyright (c) 2017 Enzo Ragucci et al. This is an open access article distributed under the Creative Commons Attribution License, which permits unrestricted use, distribution, and reproduction in any medium, provided the original work is properly cited.

\begin{abstract}
Cushing syndrome (CS), a complex, multisystemic condition resulting from prolonged exposure to cortisol, is frequently associated with nonalcoholic fatty liver disease (NAFLD). In patients with adrenal adenoma(s) and NAFLD, it is essential to rule out coexisting endocrine disorders like CS, so that the underlying condition can be properly addressed. We report a case of a 49year-old woman with a history of hypertension, prediabetes, dyslipidemia, biopsy-confirmed steatohepatitis, and benign adrenal adenoma, who was referred for endocrine work-up for persistent weight gain. Overt Cushing features were absent. Biochemical evaluation revealed nonsuppressed cortisol on multiple 1-mg dexamethasone suppression tests, suppressed adrenocorticotropic hormone, and low dehydroepiandrosterone sulfate. The patient initially declined surgery and was treated with mifepristone, a competitive glucocorticoid receptor antagonist. In addition to improvements in weight and hypertension, substantial reductions in her liver enzymes were noted, with complete normalization by 20 weeks of therapy. This case suggests that autonomous cortisol secretion from adrenal adenoma(s) could contribute to the metabolic and liver abnormalities in patients with NAFLD. In conclusion, successful management of CS with mifepristone led to marked improvement in the liver enzymes of a patient with long-standing NAFLD.
\end{abstract}

\section{Introduction}

Nonalcoholic fatty liver disease (NAFLD) is a common cause of chronic liver disease in westernized countries, affecting $17 \%$ to $30 \%$ of the population [1]. Defined histologically as the accumulation of fat within the hepatocytes that exceeds $5 \%$ of liver weight [1,2], NAFLD is generally considered the hepatic manifestation of metabolic syndrome [3]. Liver damage associated with NAFLD can range from simple steatosis to nonalcoholic steatohepatitis (NASH), which may progress to cirrhosis, liver failure, and hepatocellular carcinoma $[1,2]$. NAFLD is strongly associated with risk factors including obesity, insulin resistance, type 2 diabetes, hypertension, and dyslipidemia. Although secondary causes of NAFLD (e.g., lipid metabolism disorders, medications, and other diseases) occur in the minority of cases [1], it is important to exclude them during the differential diagnosis, especially in patients with coexisting adrenal adenoma(s).
Both hypercortisolism from exogenous sources and that from endogenous sources are recognized causes of NAFLD [3]. For instance, NAFLD is frequently seen in patients with Cushing syndrome (CS) [4], a complex, multisystemic condition resulting from prolonged exposure to cortisol. A computed tomography- (CT-) based study found hepatic steatosis in $20 \%$ of CS patients with active disease [4].

The mechanisms by which cortisol impacts lipid metabolism are complex $[5,6]$ and not yet fully elucidated. Here we report a case of autonomous cortisol secretion due to an adrenal adenoma in which medical therapy with the competitive glucocorticoid receptor antagonist mifepristone resulted in biochemical remission of NAFLD.

\section{Case Presentation}

A 49-year-old woman with a medical history of hypertension, prediabetes, dyslipidemia, and histologically confirmed 

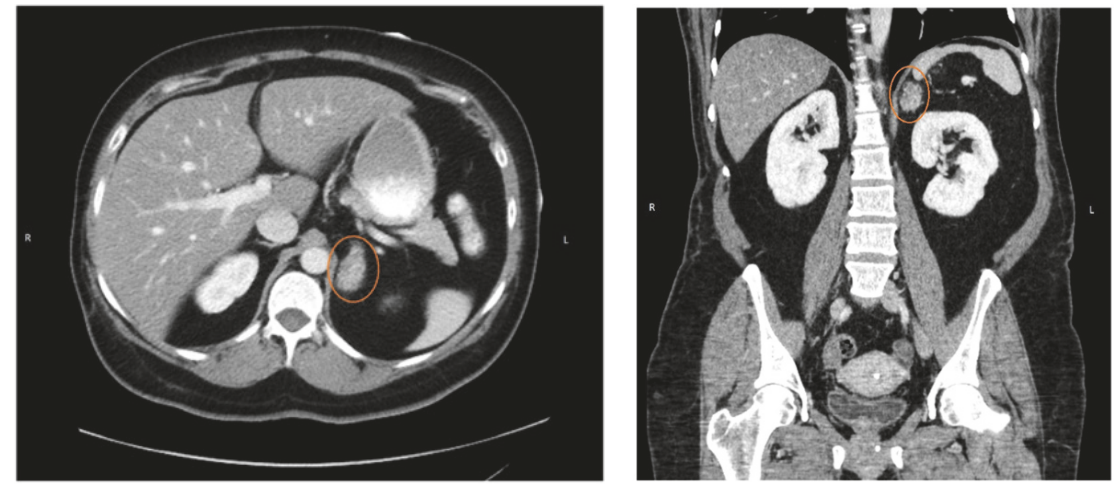

FIGURE 1: Images showing left adrenal adenoma measuring $2.9 \times 1.9 \times 2.5 \mathrm{~cm}$ (shown in circles).

NASH $(80 \%$ diffused steatosis with pericellular inflammation) was referred by her primary care provider for endocrine evaluation for complaint of persistent weight gain with central obesity. Two years prior to the referral she was discovered to have a left adrenal adenoma on a routine abdominal CT scan for evaluation of nephrolithiasis. Phenotypic features of overt CS (i.e., moon face, striae, buffalo hump, etc.) were not present, and biochemical evaluation of the adenoma was not performed at that time. At referral, the patient still did not have overt phenotypic features of CS. A follow-up scan revealed a stable benign left adrenal adenoma $(2.9 \times$ $1.9 \times 2.5 \mathrm{~cm})$ measuring 14 Hounsfield units on noncontrast CT (Figure 1). The adenoma was described as lobulated with a high fat content. Baseline clinical characteristics and laboratory findings are listed in Table 1.

Results of hormonal testing were negative for pheochromocytoma and primary aldosteronism. Testing for autonomous cortisol secretion showed mildly elevated urinary free cortisol (UFC), failure to suppress cortisol on multiple 1-mg overnight dexamethasone suppression tests (DSTs), elevated late-night salivary cortisol, suppressed adrenocorticotropic hormone (ACTH) on multiple tests, and low dehydroepiandrosterone sulfate (DHEA-S) (Table 1).

The patient declined adrenalectomy. Ketoconazole was not considered because of the patient's fatty liver and elevated liver enzymes. Medical therapy with mifepristone (Korlym ${ }^{\circledR}$, Corcept Therapeutics, Menlo Park, CA) was initiated at $300 \mathrm{mg}$ per day and increased to $900 \mathrm{mg}$ during the 34 weeks of treatment. After 4 weeks, her antihypertensive medication (amlodipine/olmesartan medoxomil 10/20 mg) was discontinued and her blood pressure remained stable (Figure 2). During the course of mifepristone treatment, she lost $16 \mathrm{lbs}$ and marked improvement in her liver enzymes was noted, with complete normalization by week 20 (Figure 3 ).

At week 20 she complained of vaginal spotting, and a pelvic ultrasound showed multiple uterine fibroids. Subsequently she elected to undergo a hysterectomy and a left adrenalectomy. Mifepristone was discontinued 2 weeks prior to the adrenalectomy. Her prediabetes remained stable during mifepristone treatment; $\mathrm{HbAlc}$ was $40 \mathrm{mmol} / \mathrm{mol}$ before surgery.
TABLE 1: Baseline patient characteristics and laboratory findings.

\begin{tabular}{lc}
\hline Parameter & Result \\
\hline Age, years & 49 \\
BMI, kg/m & 30.6 \\
BP, mmHg & $142 / 90$ \\
HbAlc, mmol/mol & 41 \\
Lipids, mmol/L & \\
$\quad$ Total cholesterol & 7.1 \\
LDL & 5.3 \\
TG & 1.5 \\
Liver function & \\
$\quad$ AST, U/L (normal 2-40) & 110 \\
$\quad$ ALT, U/L (normal 2-60) & 232 \\
Endocrine evaluation & \\
$\quad$ UFC, nmol/d (normal 11-138) & 172.8 \\
1-mg DST, nmol/L (normal < 49.5) & $364.3 ; 345.0$ \\
Late night salivary cortisol, nmol/L (normal < 2.5) & 5.2 \\
ACTH, pmol/L (normal 1.1-9.9) & $<1.1 \times 2$ \\
DHEA-S, $\mu$ mol/L (normal 1.1-7.9) & 0.54 \\
\hline
\end{tabular}

ACTH, adrenocorticotropic hormone; ALT, alanine transaminase; AST, aspartate transaminase; BMI, body mass index; BP, blood pressure; DHEA-S, dehydroepiandrosterone sulfate; DST, dexamethasone suppression test; HbAlc, glycated hemoglobin Alc; LDL, low-density lipoprotein; TG, triglyceride; UFC, urinary free cortisol.

During the 34-week course of medical therapy with mifepristone, the patient's hypothalamic-pituitary-adrenal (HPA) axis recovered, as indicated by increases in ACTH levels from undetectable at baseline and week 16 to $2.4 \mathrm{pmol} / \mathrm{L}$ at week 24 (Figure 4). She was treated postoperatively with glucocorticoid replacement (hydrocortisone $50 \mathrm{mg}$ ), which was tapered and discontinued within 6 weeks. Her liver enzymes remained within the normal range and there were no further changes in blood pressure and weight 1 year postoperatively. Her most recent HbAlc was $33 \mathrm{mmol} / \mathrm{mol}$ (5.2\%). 


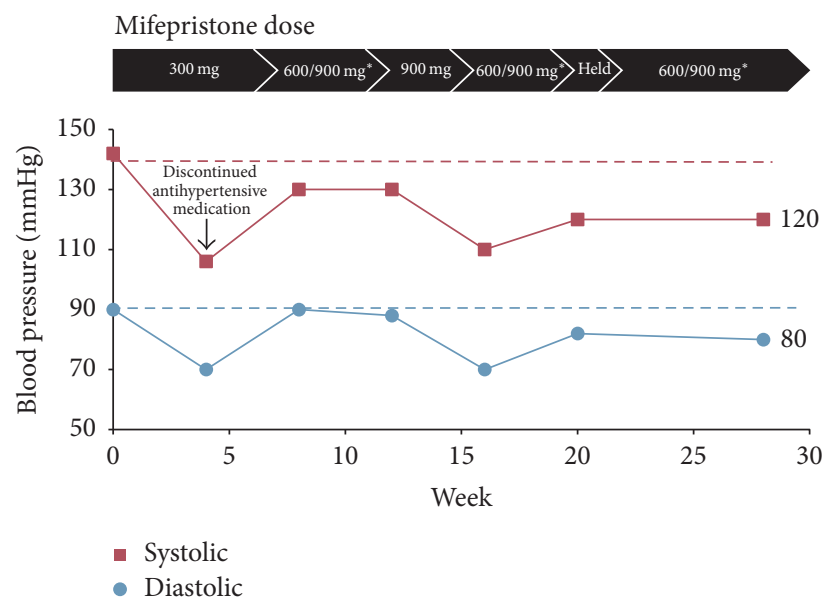

Figure 2: Change in blood pressure over time after initiating mifepristone treatment. * Alternating daily doses of 600 and $900 \mathrm{mg}$.

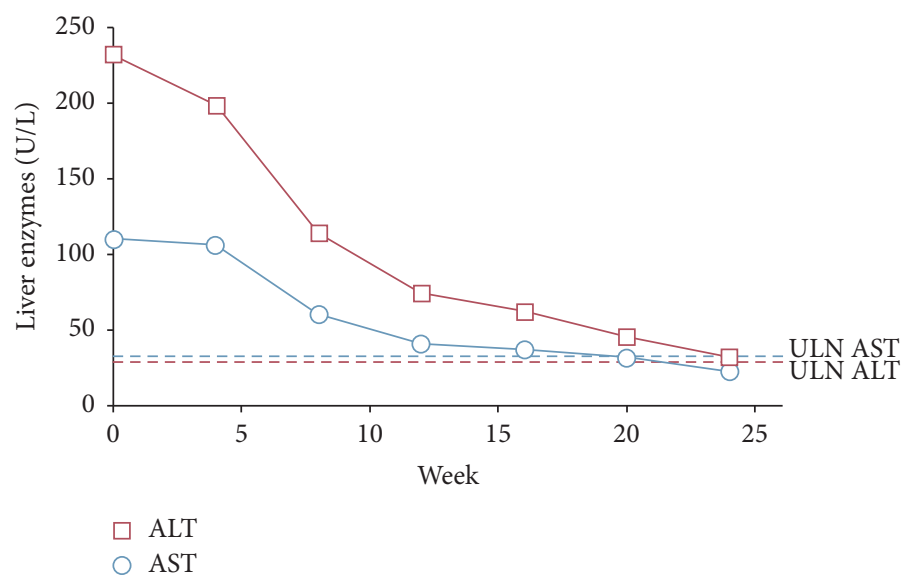

FIGURE 3: Change in liver enzymes over time after initiating mifepristone treatment. ALT, alanine transaminase; AST, aspartate transaminase; ULN, upper limit of normal.

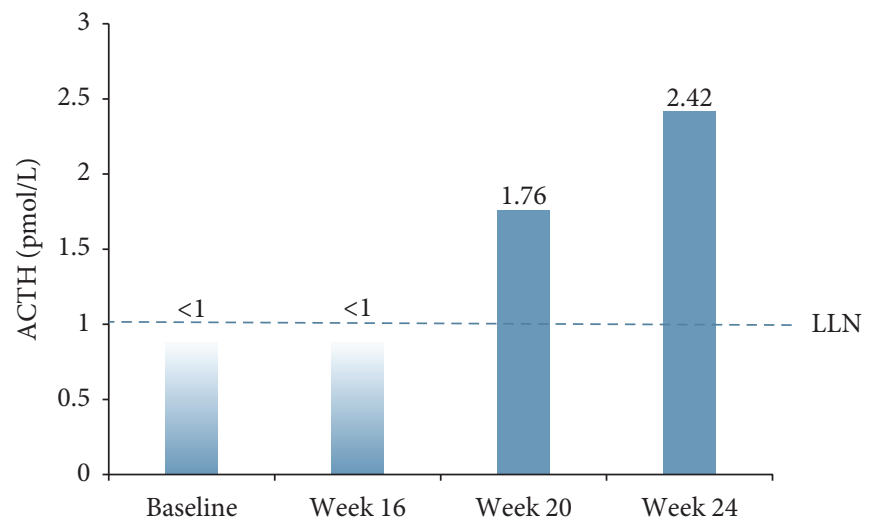

FIGURE 4: Change in ACTH over time after initiating mifepristone treatment. ACTH, adrenocorticotropic hormone; LLN, lower limit of normal. 


\section{Discussion}

We have described a case of hypercortisolism due to an adrenal adenoma associated with biopsy-confirmed NASH. The patient was treated medically with mifepristone therapy for 34 weeks. In addition to improvement in cardiometabolic parameters associated with hypercortisolism, we also noted marked improvement in her liver function tests (LFTs) during treatment.

NAFLD is associated with cardiometabolic risk factors (obesity, diabetes, dyslipidemia, and hypertension) and is diagnosed by hepatic steatosis on imaging or histology and exclusion of other causes of liver disease [7]. Although LFTs are a good surrogate biomarker for NAFLD, a substantial portion of patients, $79 \%$ according to one study [8], may have normal LFTs. Thus, liver biopsy, while being costly and invasive, remains the only definitive method for diagnosis.

NAFLD is frequently seen in patients with CS [4], which is not surprising as metabolic derangements are also common in CS. In fact, one study found a higher prevalence and greater severity of NAFLD among patients with CS when compared to matched cohorts of nonfunctioning adrenal adenomas and controls [9]. A high prevalence of metabolic syndrome features and cardiometabolic risk factors has also been reported in patients with adrenal adenoma(s) [10-12]. Other studies have shown HPA axis dysfunction among patients with NAFLD [13-15]. In overweight/obese NAFLD patients, HPA axis dysfunction was correlated with the severity of NAFLD [16]. Together, these studies suggest that subtle and chronic activation of the HPA axis can contribute to the development and progression of NAFLD.

Hypercortisolism without classically described features of overt CS occurs in up to $30 \%$ of patients with adrenal adenomas [17]. Clinical assessment for signs and symptoms of hypercortisolism, as well as biochemical evaluation using the 1-mg overnight DST, is recommended as part of the workup for patients with newly discovered adrenal adenomas [18]. In our case, the patient was not referred for endocrine evaluation until 2 years after the adenoma was discovered and her comorbidities had worsened. This case underscores the need for greater awareness among primary care providers of the importance of cortisol evaluation for benign adrenal adenomas, regardless of clinical symptomology.

Mifepristone, a competitive glucocorticoid receptor antagonist, is an effective medical therapy for patients with endogenous CS [19]. Patients from the SEISMIC trial demonstrated significant improvement in glucose abnormalities (assessed by oral glucose tolerance test), significant reductions in fasting plasma glucose and HbAlc, and a rapid and significant decrease in mean area under the curve insulin levels [19]. Together, these suggest improved insulin sensitivity.

The effects of mifepristone on metabolic parameters in patient populations outside of CS have also been explored. In a small study of patients with type 2 diabetes and fatty liver, short-term doses of mifepristone and metyrapone, a cortisol biosynthesis inhibitor, appeared to improve insulin sensitivity [10]. Two other clinical studies conducted in healthy men demonstrated that mifepristone significantly attenuated not only the side effect of weight gain caused by second-generation antipsychotic medications, but also the increases in fasting plasma insulin and triglyceride levels caused by their use $[20,21]$.

In our patient with hypercortisolism and biopsy-confirmed NASH, mifepristone treatment was associated with dramatic improvement in LFTs, as well as improvements in weight and hypertension leading to discontinuation of her antihypertensive medication. Follow-up liver imaging was not available for comparison; however, the overall improved functional and metabolic status of the patient, along with no signs or symptoms of deteriorating liver function (cirrhosis), support the LFT findings. The effect of mifepristone on LFTs was a novel finding and suggests that mifepristone may offer an alternative treatment to surgery in patients with hypercortisolism and NAFLD. It also may provide a useful method to assess the contribution of autonomous cortisol secretion of an adrenal adenoma to the cardiometabolic profile and liver abnormalities in patients with NAFLD. Larger prospective studies are needed to determine whether mifepristone use in patients with NAFLD and adrenal adenoma(s) can be used as a screening tool to select patients who will benefit from adrenalectomy.

\section{Disclosure}

Data from this case report were presented at the annual ENDO meeting in San Diego, CA, on March 5-8, 2015.

\section{Conflicts of Interest}

Drs. Moraitis, Nguyen, and Ms. Lamerson are employees of Corcept Therapeutics. Dr. Ragucci has no conflicts of interest to disclose.

\section{Acknowledgments}

The authors wish to thank Sarah Mizne, PharmD, of MedVal Scientific Information Services, LLC, for providing professional editorial assistance. Funding to support the preparation of this manuscript was provided to MedVal by Corcept Therapeutics. This manuscript was prepared according to the International Society for Medical Publication Professionals' "Good Publication Practice for Communicating CompanySponsored Medical Research: the GPP3 Guidelines" and the International Committee of Medical Journal Editors' "Uniform Requirements for Manuscripts Submitted to Biomedical Journals."

\section{References}

[1] J. M. Kneeman, J. Misdraji, and K. E. Corey, "Secondary causes of nonalcoholic fatty liver disease," Therapeutic Advances in Gastroenterology, vol. 5, no. 3, pp. 199-207, 2012.

[2] P. Angulo, "GI epidemiology: nonalcoholic fatty liver disease," Alimentary Pharmacology \& Therapeutics, vol. 25, no. 8, pp. 883-889, 2007.

[3] F. Ferraù and M. Korbonits, "Metabolic comorbidities in Cushing's syndrome," European Journal of Endocrinology, vol. 173, no. 4, pp. M133-M157, 2015. 
[4] A. G. Rockall, S. A. Sohaib, D. Evans et al., "Hepatic steatosis in Cushing's syndrome: a radiological assessment using computed tomography," European Journal of Endocrinology, vol. 149, no. 6, pp. 543-548, 2003.

[5] C. P. Woods, J. M. Hazlehurst, and J. W. Tomlinson, "Glucocorticoids and non-alcoholic fatty liver disease," The Journal of Steroid Biochemistry and Molecular Biology, vol. 154, article no. 4458, pp. 94-103, 2015.

[6] L. L. Gathercole, S. A. Morgan, I. J. Bujalska, D. Hauton, P. M. Stewart, and J. W. Tomlinson, "Regulation of lipogenesis by glucocorticoids and insulin in human adipose tissue," PLoS ONE, vol. 6, no. 10, Article ID e26223, 2011.

[7] N. Chalasani, Z. Younossi, and J. E. Lavine, "The diagnosis and management of non-alcoholic fatty liver disease: practice guideline by the American Association for the Study of Liver Diseases, American College of Gastroenterology, and the American Gastroenterological Association," Hepatology, vol. 55, no. 6, pp. 2005-2023, 2012.

[8] J. D. Browning, L. S. Szczepaniak, R. Dobbins et al., "Prevalence of hepatic steatosis in an urban population in the United States: impact of ethnicity," Hepatology, vol. 40, no. 6, pp. 1387-1395, 2004.

[9] S. Yener, A. Comlekci, S. Ertilav, M. Secil, M. Akarsu, and S. Yesilli, "Non-alcoholic fatty liver disease in subjects with nonfunctioning adrenal adenomas," Turkish Journal of Endocrinology and Metabolism, vol. 15, pp. 116-120, 2011.

[10] D. P. Macfarlane, P. J. Raubenheimer, T. Preston et al., "Effects of acute glucocorticoid blockade on metabolic dysfunction in patients with type 2 diabetes with and without fatty liver," American Journal of Physiology-Gastrointestinal and Liver Physiology, vol. 307, no. 7, pp. G760-G768, 2014.

[11] R. Rossi, L. Tauchmanova, A. Luciano et al., "Subclinical Cushing's syndrome in patients with adrenal incidentaloma: clinical and biochemical features," The Journal of Clinical Endocrinology \& Metabolism, vol. 85, no. 4, pp. 1440-1448, 2000.

[12] M. Terzolo, A. Pia, A. Alì et al., "Adrenal incidentaloma: a new cause of the metabolic syndrome?" The Journal of Clinical Endocrinology \& Metabolism, vol. 87, no. 3, pp. 998-1003, 2002.

[13] G. Zoppini, G. Targher, C. Venturi, C. Zamboni, and M. Muggeo, "Relationship of nonalcoholic hepatic steatosis to overnight low-dose dexamethasone suppression test in obese individuals," Clinical Endocrinology, vol. 61, no. 6, pp. 711-715, 2004.

[14] J. Westerbacka, H. Yki-Järvinen, S. Vehkavaara et al., "Body fat distribution and cortisol metabolism in healthy men: enhanced $5 \beta$-reductase and lower cortisol/cortisone metabolite ratios in men with fatty liver," The Journal of Clinical Endocrinology \& Metabolism, vol. 88, no. 10, pp. 4924-4931, 2003.

[15] G. Targher, L. Bertolini, G. Zoppini, L. Zenari, and G. Falezza, "Relationship of non-alcoholic hepatic steatosis to cortisol secretion in diet-controlled Type 2 diabetic patients," Diabetic Medicine, vol. 22, no. 9, pp. 1146-1150, 2005.

[16] G. Targher, L. Bertolini, S. Rodella, G. Zoppini, L. Zenari, and G. Falezza, "Associations between liver histology and cortisol secretion in subjects with nonalcoholic fatty liver disease," Clinical Endocrinology, vol. 64, no. 3, pp. 337-341, 2006.

[17] I. Chiodini, "Clinical review: diagnosis and treatment of subclinical hypercortisolism," The Journal of Clinical Endocrinology \& Metabolism, vol. 96, no. 5, pp. 1223-1236, 2011.

[18] M. Fassnacht, W. Arlt, I. Bancos et al., "Management of adrenal incidentalomas: European Society of Endocrinology
Clinical Practice Guideline in collaboration with the European Network for the Study of Adrenal Tumors," European Journal of Endocrinology, vol. 175, no. 2, pp. G1-G34, 2016.

[19] M. Fleseriu, B. M. K. Biller, J. W. Findling, M. E. Molitch, D. E. Schteingart, and C. Gross, "Mifepristone, a glucocorticoid receptor antagonist, produces clinical and metabolic benefits in patients with Cushing's syndrome," The Journal of Clinical Endocrinology \& Metabolism, vol. 97, no. 6, pp. 2039-2049, 2012.

[20] C. Gross, C. M. Blasey, R. L. Roe, K. Allen, T. S. Block, and J. K. Belanoff, "Mifepristone treatment of olanzapine-induced weight gain in healthy men," Advances in Therapy, vol. 26, no. 10, pp. 959-969, 2009.

[21] C. Gross, C. M. Blasey, R. L. Roe, and J. K. Belanoff, "Mifepristone reduces weight gain and improves metabolic abnormalities associated with risperidone treatment in normal men," Obesity, vol. 18, no. 12, pp. 2295-2300, 2010. 


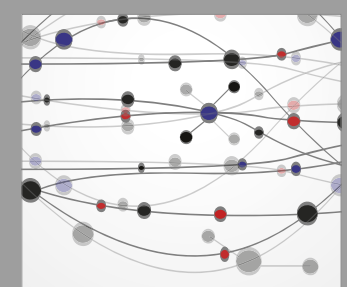

The Scientific World Journal
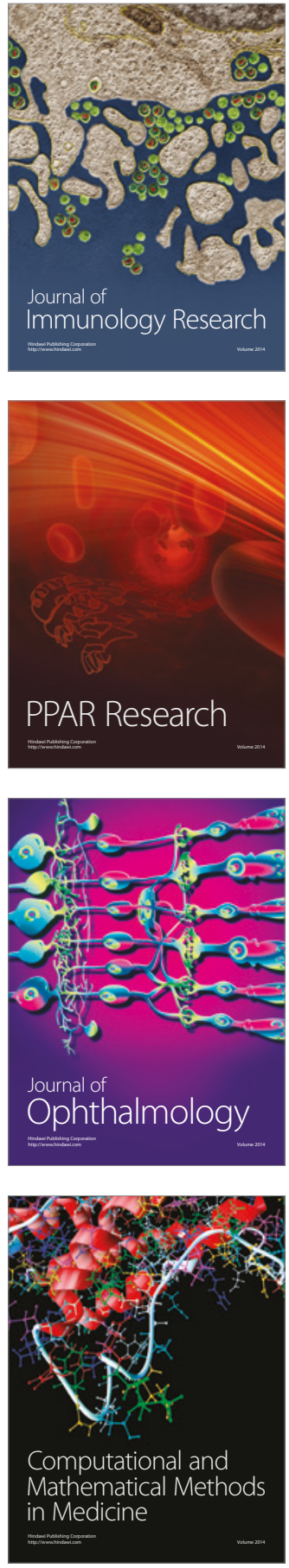

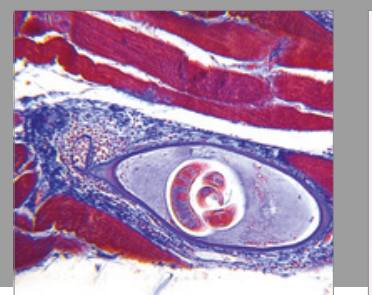

Gastroenterology Research and Practice
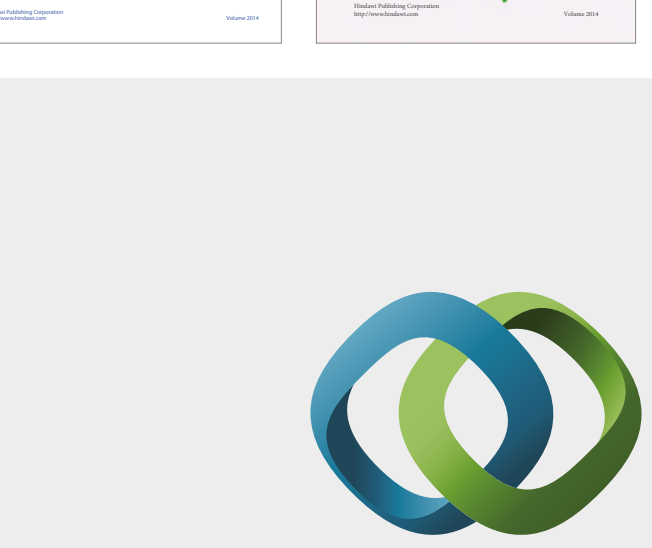

\section{Hindawi}

Submit your manuscripts at

https://www.hindawi.com
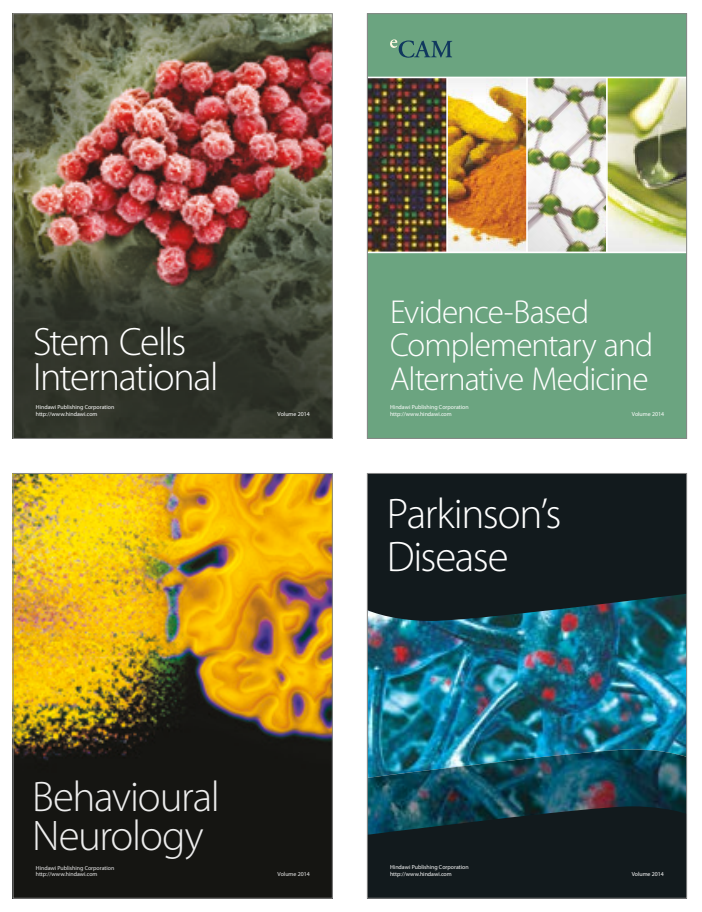
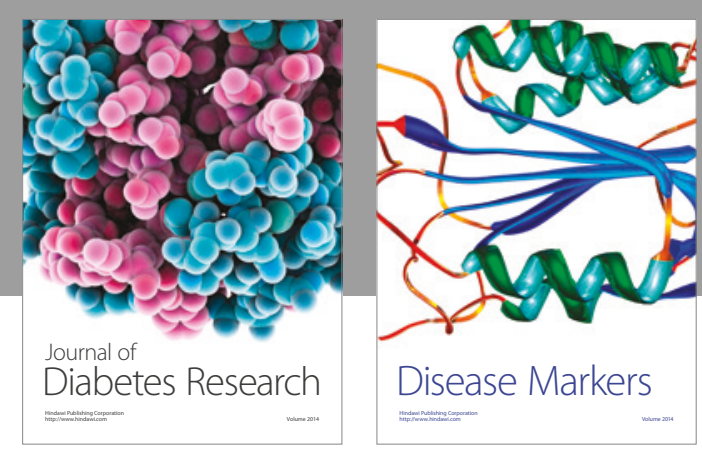

Disease Markers
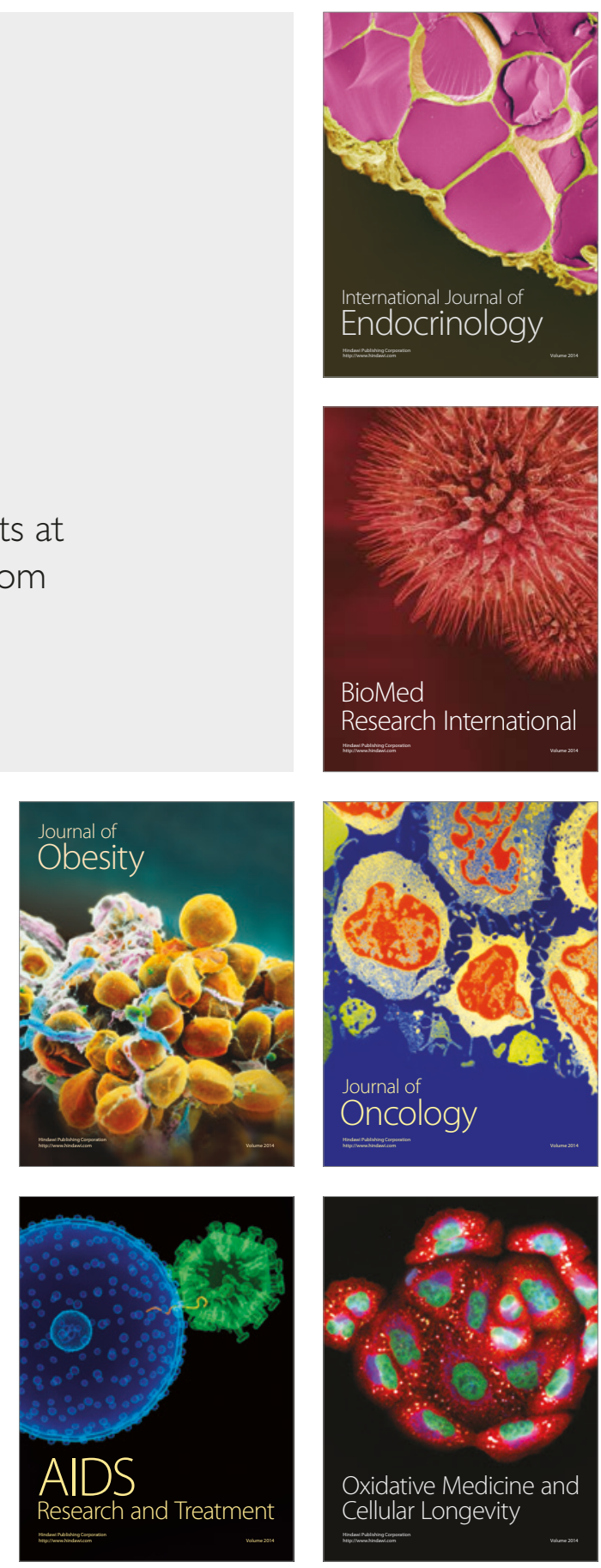